\title{
Reliability and Clinical Relevance of the HIV-1 Drug Resistance Test in Patients With Low Viremia Levels
}

Maria Mercedes Santoro, ${ }^{1}$ Lavinia Fabeni, ${ }^{2}$ Daniele Armenia, ${ }^{1}$ Claudia Alteri, ${ }^{1}$ Domenico Di Pinto, ${ }^{1}$ Federica Forbici, ${ }^{2}$ Ada Bertoli, ${ }^{1,3}$ Domenico Di Carlo, ${ }^{1}$ Caterina Gori, ${ }^{2}$ Stefania Carta, ${ }^{2}$ Valentina Fedele, ${ }^{2}$ Roberta D'Arrigo, ${ }^{2}$ Giulia Berno, ${ }^{2}$ Adriana Ammassari, ${ }^{2}$ Carmela Pinnettii, ${ }^{2}$ Emanuele Nicastri, ${ }^{2}$ Alessandra Latini, ${ }^{4}$ Chiara Tommasi, ${ }^{2}$ Evangelo Boumis, ${ }^{2}$ Nicola Petrosillo, ${ }^{2}$ Gianpiero D'0ffizi, ${ }^{2}$ Massimo Andreoni, ${ }^{1,3}$ Francesca Ceccherini-Silberstein, ${ }^{1}$ Andrea Antinori, ${ }^{2}$ and Carlo Federico Perno ${ }^{1,2,3}$

${ }^{1}$ University of Rome Tor Vergata; ${ }^{2}$ L. Spallanzani Hospital; ${ }^{3}$ University Hospital Tor Vergata; and ${ }^{4}$ San Gallicano Hospital, Rome, Italy

(See the Major Article by Gonzalez-Serna et al on pages 1165-73, and the Editorial Commentary by Richman on pages 1174-5.)

Background. We evaluated reliability and clinical usefulness of genotypic resistance testing (GRT) in patients for whom combination antiretroviral therapy (cART) was unsuccessful with viremia levels $50-1000$ copies $/ \mathrm{mL}$, for whom GRT is generally not recommended by current guidelines.

Methods. The genotyping success rate was evaluated in 12828 human immunodeficiency virus type 1 (HIV-1) plasma samples with viremia $>50$ copies $/ \mathrm{mL}$, tested using the commercial ViroSeq HIV-1 Genotyping System or a homemade system. Phylogenetic analysis was performed to test the reliability and reproducibility of the GRT at low-level viremia (LLV). Drug resistance was evaluated in 3895 samples from 2200 patients for whom treatment was unsuccessful (viremia $>50$ copies $/ \mathrm{mL}$ ) by considering the resistance mutations paneled in the 2013 International Antiviral Society list.

Results. Overall, the success rate of amplification/sequencing was 96.4\%. Viremia levels of 50-200 and 201-500 copies $/ \mathrm{mL}$ afforded success rates of $67.2 \%$ and $88.1 \%$, respectively, reaching $93.2 \%$ at $501-1000$ copies $/ \mathrm{mL}$ and $\geq 97.3 \%$ above 1000 copies $/ \mathrm{mL}$. A high homology among sequences belonging to the same subject for $96.4 \%$ of patients analyzed was found. The overall resistance prevalence was $74 \%$. Drug resistance was commonly found also at LLV. In particular, by stratifying for different viremia ranges, detection of resistance was as follows: 50-200 copies $/ \mathrm{mL}=52.8 \% ; 201-500=70 \%$; $501-1000=74 \% ; 1001-10000=86.1 \% ; 10001-100000=76.7 \%$; and $>100000=63 \%(P<.001)$. Similar bell-shaped results were found when the GRT analysis was restricted to 2008-2012, although at a slightly lower prevalence.

Conclusions. In patients failing cART with LLV, HIV-1 genotyping provides reliable and reproducible results that are informative about emerging drug resistance.

Keywords. HIV-1 genotyping; HIV-1 low viremia; drug resistance; phylogenesis; clinical outcome.

Over the past 15 years, antiretroviral therapy for the treatment of human immunodeficiency virus type 1 (HIV-1) infection has improved; to date, about $90 \%$ of HIV-1-infected patients who start a first-line regimen

Received 13 August 2013; accepted 2 November 2013; electronically published 14 January 2014

Presented in part: 11th European Meeting on HIV and Hepatitis Treatment and Strategies and Antiviral Drug Resistance, Rome, Italy, 20-22 March 2013. Abstract 0_23; and the XX International Workshop on HIV and Hepatitis Virus Drug Resistance and Curative Strategies, Toronto, Canada, 4-8 June 2013. Antiviral Therapy 2013; 18(suppl 1):A128.

Correspondence: Carlo Federico Perno, PhD, MD, Department of Experimental Medicine and Surgery, University of Rome Tor Vergata, Via Montpellier 1, Rome 00133, Italy (cf.perno@uniroma2.it). achieve virologic suppression [1-10]. However, therapy failures are still observed in clinical practice; particularly at early time points, many are characterized by low-level viremia (LLV). Standard-of-care management recommends use of resistance testing to guide further therapy. One area of uncertainty is the evaluation of treatment failure in patients with LLV. Treatment guidelines usually do not recommend genotypic resis-

Clinical Infectious Diseases 2014;58(8):1156-64

(C) The Author 2014. Published by Oxford University Press on behalf of the Infectious Diseases Society of America. All rights reserved. For Permissions, please e-mail: journals.permissions@oup.com.

DOI: 10.1093/cid/ciu020 
tance testing (GRT) for plasma HIV RNA <500-1000 copies/ $\mathrm{mL}$. This potential limitation of GRT mostly derives from the detection limits of commercial assays, as well as by the technical difficulty of many laboratories in obtaining consistent results with such LLV, yet some studies support the use of GRT, and laboratories increasingly report success in performing genotypes at this level [11-26].

In this study, we provide data to support reliability and usefulness of GRT at viremia levels $\leq 500-1000$ copies/mL by analyzing a large population of HIV-1-infected patients followed in central Italy who underwent GRT in routine clinical practice. Moreover, we evaluated whether different viremia levels affect the detection of drug resistance in HIV-1-infected patients who failed therapy.

\section{MATERIALS AND METHODS}

\section{Patients}

This retrospective study included $13926 \mathrm{HIV}-1$ plasma samples that were genotyped during 1999-2012 in 2 clinical centers in Rome (Italy) for routine clinical purposes. Sample information (date of sampling, final results of sequencing, nucleotide sequences obtained, mutations found in each sequence), together with the data of patients for whom genotyping was performed (ie, viroimmunologic, clinical, and therapeutic data) were recorded in an anonymous database.

For each sample, viremia value at genotyping was known. We focused our analyses on samples with viremia $>50$ copies $/ \mathrm{mL}$ $(\mathrm{N}=12828)$ that were stratified in 6 groups according to different viremia ranks (copies/mL): 50-200, 201-500, 501-1000, 1001-10 000, $10001-100000$, and >100 000 .

\section{HIV-1 RNA Load}

Depending on methodologies available during 1999-2012, plasma viremia was determined using 3 different assays: the bDNA version 3.0 (until January 2009; Bayer Corporation, Diagnostics Division, Tarrytown, New York), the Abbott RealTime HIV-1 (February 2009-February 2012; Abbott, Chicago, Illinois) and the Roche Cobas CA/CTM version 2.0 (starting from March 2012; Roche, Mannheim, Germany). These assays quantify HIV-1 RNA in the range of 50-500 000 copies $/ \mathrm{mL}, 40$ 10000000 copies/mL, and 20-10 000000 copies/mL, respectively. Previous studies demonstrated the results obtained by these assays to be well correlated, with a difference of $>0.5$ $\log _{10}$ copies/mL, only for a few samples [27-29].

\section{HIV-1 pol Sequencing}

HIV-1 genotype analysis was performed on plasma samples by using either the ViroSeq HIV-1 genotyping system (Abbott Molecular) and/or a homemade system, designed to improve the performance of the ViroSeq system itself [30]. Indeed, genotyping success by this commercial kit is generally guaranteed for samples with viremia $\geq 2000$ copies/mL $[31,32]$. Therefore, some steps of the ViroSeq system were modified, to also test HIV-1 pol sequences in subjects with viremia $<2000$ copies $/ \mathrm{mL}$. All of the details on the amplification and sequencing procedures can be found in the Supplementary Methods and Supplementary Figure 1.

\section{Subtyping Analysis}

All HIV-1 pol sequences were aligned in Bio-Edit and compared to reference sequences for major HIV-1 subtypes and circular recombinant forms (CRFs), available at the Los Alamos database (http://www.hiv.lanl.gov); a phylogenetic tree was performed. To analyze trends in subtype genetic diversity over time, genetic distances were calculated by using the maximum-likelihood method in MEGA (http://www.megasoftware. net/), by using Kimura 2-parameter model as the best-fitting evolution model for tree reconstruction [33]. The tree was shown by using the graphical user interface FigTree. Subtype classification was confirmed also by the REGA subtype tool (http://www.bioafrica.net/rega-genotype/html/subtypinghiv. html), the COMET subtype tool (http://comet.retrovirology.lu/), and the DataMonkey subtype tool (http://www.datamonkey. org/dataupload_scueal.php). To improve the accuracy of recombinant and unique forms, RDP3 software (http://web.cbio. uct.ac.za/ darren/rdp.html) and Splits Tree software (http://en. bio-soft.net/tree/SplitsTree.html) were used.

\section{Evaluation of Genotypic Success Rate and Genotyping Reliability}

Genotyping success rate was determined on the overall population and according to the different viremia ranks (50-200, 201-500, 501-1000, 1001-10 000, $10001-100000$, and >100 000 copies/ $\mathrm{mL}$ ), regardless of the genotyping platform upgrades (equipment, kits, and reagents) that occurred from 1999 to 2012.

To ensure that there was no cross-contamination of samples analyzed and to test genotyping reliability for samples with viremia $\leq 500$ copies/mL, a phylogenetic analysis was performed on a subgroup of 1613 pol sequences, obtained from 470 patients with at least 1 GRT performed on samples with viremia $\leq 500$ copies/mL and at least 1 GRT with viremia >1000 copies/ $\mathrm{mL}$. The phylogenetic analysis of pol sequences was performed by using the Kimura 2-parameter model of MEGA version 5.05, with the same parameters as previously described [33].

\section{Evaluation of Resistance in Patients Who Had Failed Therapy}

The prevalence of drug resistance was evaluated, and stratified according to different viremia levels, in a subset of 3895 samples successfully genotyped from 2200 patients with complete therapeutic history, for whom a GRT was required because of virologic failure (defined as viremia $>50$ copies $/ \mathrm{mL}$ ). Resistance to an antiretroviral drug class was defined by the presence of at least 1 primary resistance mutation (PRM) included in the mutation list paneled by the International Antiviral Society in 2013 
[34], considering the nucleos(t)ide reverse transcriptase inhibitors (NRTIs), nonnucleos(t)ide reverse transcriptase inhibitors (NNRTIs), and protease inhibitors (PIs). In particular, we have defined $(i)$ the resistance to any drug class in the overall samples analyzed; (ii) the resistance to NRTIs among samples from patients who received regimens that contained NRTIs; ( $i i i)$ the resistance to NNRTIs among samples from patients who received regimens that contained NNRTIs; (iv) the resistance to PIs among samples from patients who received regimens that contained ritonavir-boosted PIs (PI/r); and ( $v$ ) the resistance to PIs among samples from patients who received regimens that contained ritonavir-unboosted PIs.

To better understand the clinical relevance of GRT in patients failing with LLV at the time of modern anti-HIV therapies, the prevalence of single PRMs was also evaluated on the 1317 samples from patients for whom a GRT was required because of virologic failure in the years 2008-2012. This analysis was performed by dividing the samples into 2 groups according to viremia levels $\leq 1000$ copies/mL $(n=436)$ or $>1000$ copies/mL $(n=881)$.

\section{Patient Outcome Analysis}

To evaluate the effect of LLV resistance on subsequent virologic outcome, further analyses were restricted to 51 previously drugnaive patients on first-line regimens for whom a GRT was requested at viremia levels of $50-1000$ copies/mL. Patients were included only if they were followed as long as they were receiving constant therapy without any changes or interruptions.

\section{Statistical Analysis}

Potential differences among the different viremia groups were evaluated as follows: $(i)$ for the categorical variables, by the $\chi^{2}$ test for trend (to compare all viremia groups) and Pearson $\chi^{2}$ test or Fisher exact test when expected frequencies were $<5$ (to compare 2 viremia groups at a time); and (ii) for the continuous variables, by the Kruskal-Wallis test (to compare all viremia groups). Regarding the virologic outcome, Kaplan-Meier analysis was used to evaluate the probability of reaching viremia $>1000$ copies/mL after LLV.

In all the analyses performed, $P<.05$ was considered as statistically significant. The statistical programs used were $\mathrm{R}$ open source software (version 2.15.1) and SPSS version 19 for Windows (SPSS Inc, Chicago, Illinois).

\section{RESULTS}

\section{Study Population}

Table 1 shows the characteristics of 12828 of 13926 plasma samples with viremia $>50$ copies $/ \mathrm{mL}$, processed for genotyping in routine clinical practice from 1999 to 2012. Among them, 4861 (37.9\%) were obtained from 4111 drug-naive patients, and 7967 (62.1\%) from 3841 drug-experienced patients.
Table 1. Characteristics of Plasma Samples With HIV-1 RNA $>50$ Copies/mL at Genotypic Resistance Testing

\begin{tabular}{|c|c|}
\hline Variable & No. (\%) \\
\hline \multicolumn{2}{|l|}{ Patients ( $N=7518$ ) } \\
\hline Patients with only 1 sample & $4950(65.8)$ \\
\hline Patients with $>1$ sample & $2568(34.2)$ \\
\hline \multicolumn{2}{|l|}{ Samples $(N=12828)$} \\
\hline From drug-naive patients & 4861 (37.9) \\
\hline From drug-experienced patients & $7967(62.1)$ \\
\hline \multicolumn{2}{|c|}{ Samples with subtype information available ${ }^{a}$} \\
\hline B & $10212(80.1)$ \\
\hline CRF02_AG & $598(4.7)$ \\
\hline $\mathrm{C}$ & $545(4.3)$ \\
\hline $\mathrm{F}$ & 422 (3.3) \\
\hline $\mathrm{BF}^{\mathrm{b}}$ & $312(2.4)$ \\
\hline G & $173(1.4)$ \\
\hline A & $157(1.2)$ \\
\hline Other & $326(2.6)$ \\
\hline \multicolumn{2}{|c|}{ Samples according to viremia ranges, copies $/ \mathrm{mL}$} \\
\hline $50-200$ & $769(6.0)$ \\
\hline $201-500$ & 489 (3.8) \\
\hline $501-1000$ & $444(3.4)$ \\
\hline $1001-10000$ & $2435(19.0)$ \\
\hline $10001-100000$ & 4845 (37.8) \\
\hline$>100000$ & $3846(30.0)$ \\
\hline
\end{tabular}

Among drug-experienced patients, viremia levels of 50-1000 copies/mL accounted for 19.2\% (1535/7967) of total genotypic requests (Figure 1). This prevalence significantly increased over time, from $1.5 \%$ in $1999-2001$ to $28.4 \%$ in $2012(P<.001)$. A consistent proportion of samples with LLV was with viremia 50-500 copies/mL (1158/1535 [75.4\%] vs 377 [24.6\%] with viremia 501-1000 copies/mL).

Phylogenetic analysis revealed that the B subtype was the most prevalent strain $(80.1 \%)$. All the other subtypes were present with a prevalence $<5 \%$; the most prevalent ones were the recombinant form CRF02_AG (4.7\%) and the subtypes C (4.3\%) and $\mathrm{F}(3.3 \%)$.

\section{Genotyping Success Rate}

The overall success rate of genotype amplification and sequencing was $96.4 \%$. The rate of success was $93.2 \%$ for samples with viremia levels $501-1000$ copies/mL, $88.1 \%$ for those with viremia 201-500 copies/mL, and decreased to a still-relevant $67.2 \%$ for viremia $50-200$ copies/mL (Table 2). The genotyping success rate was independent of subtype in all viremia groups (Table 2). By focusing the attention on the 3 most prevalent 


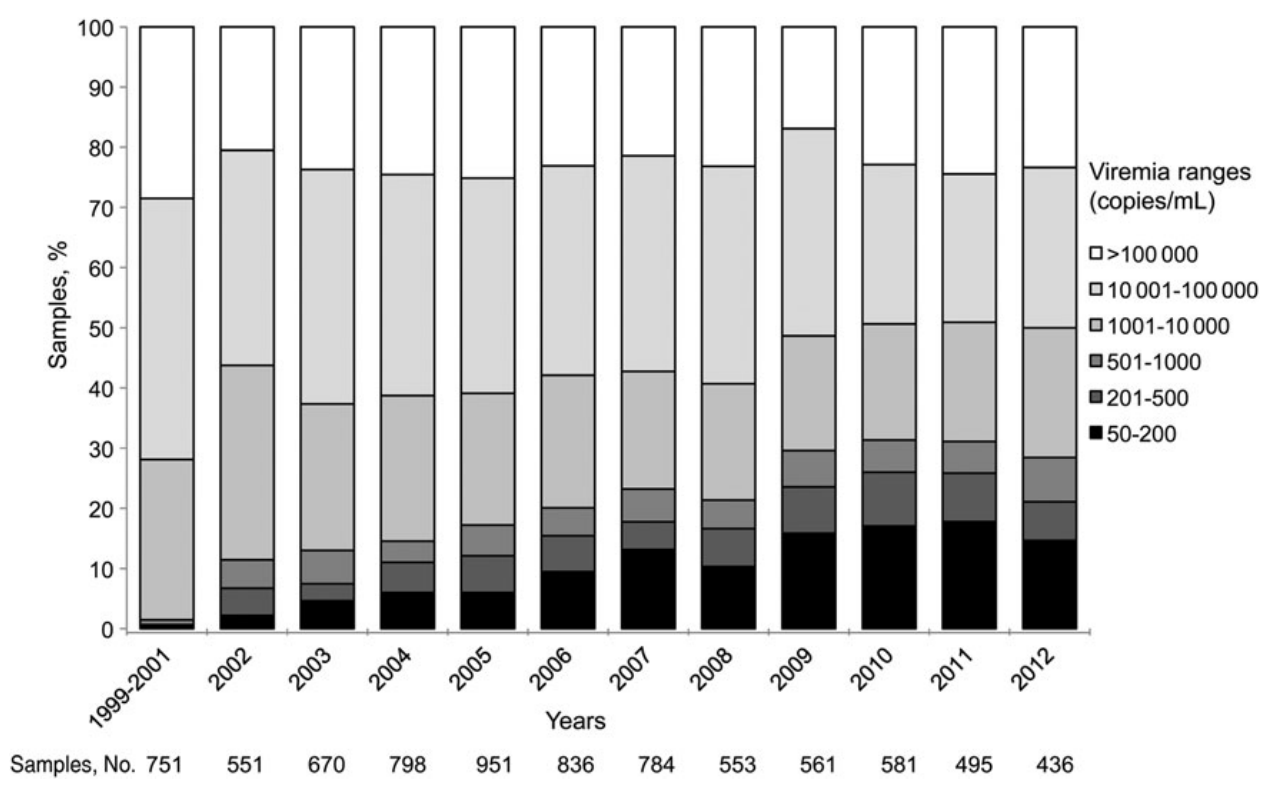

Figure 1. Genotypic requests for 7967 plasma samples from drug experienced patients, 1999-2012. The proportions of genotypic requests stratified by different viremia ranges are represented in different shades from black to white. The differences of genotypic requests over the years in patients with viremia levels $50-1000$ copies/mL vs patients with viremia levels $>1000$ copies/mL were evaluated by $\chi^{2}$ test for trend. $P<.05$ was considered significant.

non-B subtypes analyzed (C, F, CRF02_AG), no differences in the success rate were found (data not shown).

Interestingly, the additional use of a nested polymerase chain reaction (PCR) (or modified amplification protocol; see Supplementary Methods and Supplementary Figure 1) has

Table 2. HIV-1 Genotyping Resistance Success Rate According to Different Viremia Levels

\begin{tabular}{|c|c|c|c|c|}
\hline \multirow[b]{2}{*}{$\begin{array}{l}\text { Viremia Ranges, } \\
\text { Copies/mL }\end{array}$} & \multirow[b]{2}{*}{$\begin{array}{l}\text { Overall } \\
\text { No. (\% } \\
\text { Success) }\end{array}$} & \multicolumn{3}{|c|}{ Subtype } \\
\hline & & $\begin{array}{c}\text { B, No. } \\
\text { (\% Success) }\end{array}$ & $\begin{array}{c}\text { Non-B, } \\
\text { No. (\% } \\
\text { Success) }\end{array}$ & $\begin{array}{c}P \\
\text { Value }\end{array}$ \\
\hline Overall & $12828(96.4)$ & 10212 (97.1) & $2533(96.1)$ & .683 \\
\hline 50-200 & 769 (67.2) & 583 (72.9) & $139(66.2)$ & .115 \\
\hline $201-500$ & 489 (88.1) & $369(90.0)$ & $113(86.7)$ & .330 \\
\hline 501-1000 & 444 (93.2) & 329 (94.5) & $112(92.0)$ & .328 \\
\hline $1001-10000$ & 2435 (97.3) & 1967 (98.0) & 456 (96.9) & .153 \\
\hline $10001-100000$ & 4845 (99.2) & 3969 (99.3) & 867 (99.2) & .812 \\
\hline$>100000$ & 3846 (99.5) & 2995 (99.6) & 846 (99.2) & .159 \\
\hline
\end{tabular}

Abbreviation: HIV-1, human immunodeficiency virus type 1.

The success of the genotypic resistance test in plasma samples from HIV1-infected patients was evaluated on the overall population with viremia $>50$ copies $/ \mathrm{mL}(\mathrm{N}=12828$ ) and according to subtype ( $\mathrm{B}$ vs non-B), by stratifying for viremia ranges. The rate of genotyping success in patients with viremia $<50$ copies $/ \mathrm{mL}$ was $17.5 \%$.

${ }^{a}$ Potential differences in the rate of genotypic success in B and non-B subtypes were evaluated by $\chi^{2}$ test (corrected for the population size, as appropriate) or Fisher exact test, as appropriate. $P<.05$ was considered statistically significant. significantly improved the overall success rate in samples with LLV $(P<.001)$. In particular, the nested amplification contributed to $60.4 \%, 55.3 \%$, and $44.0 \%$ of the total genotypic successes with viremia levels 50-200, 201-500, and 501-1000 copies/mL, respectively. In samples with viremia levels $>1000$ copies $/ \mathrm{mL}$, the contribution of nested amplification was less relevant (from $19.2 \%$ to $3.6 \%$; data not shown).

\section{Genotyping Reliability}

To test genotyping reliability for samples with VL $\leq 1000$ copies/mL, we performed phylogenetic analysis on 1613 sequences from 470 patients having at least 1 genotypic sample with viremia 50-1000 copies/mL and at least another with viremia $>1000$ copies/mL. By evaluating each cluster, we found that sequences belonging to the same subject showed a high homology (bootstrap value $>90 \%$ ) in $96.4 \%$ of cases (453/470 patients) (Supplementary Figure 2). Only 25 of 1613 sequences (1.5\%) of the remaining 17 patients did not properly cluster within the same subject.

\section{Evaluation of Resistance According to Different Viremia Ranges in Patients Failing Therapy}

Prevalence of PRMs was analyzed on 3895 samples from a subgroup of 2200 patients at therapy failure. Patient characteristics of this subgroup are reported in Supplementary Table 1. Overall, the median year of genotyping was 2006 (interquartile range, 2003-2009), and the proportion of samples from subtype B infected-patients was about $86 \%$. 
The overall prevalence of samples with at least 1 PRM was 74\% (Table 3). PI resistance in patients treated with $\mathrm{PI} / \mathrm{r}$ was in general less frequent than NRTI or NNRTI resistance (40.5\%, vs 66\% and 77.7\%; $P<.001$; Table 3).

If we consider PI resistance only in patients for whom a firstline regimen containing a $\mathrm{PI} / \mathrm{r}$ was unsuccessful, the rate of resistance dropped dramatically to $3.7 \%$. By contrast, PI resistance in patients treated with unboosted PIs was more similar to that of those treated with an NRTI/NNRTI (61.7\%) and remained high also among patients tested at first-line failure (46.6\%).

The prevalence of resistance varied significantly by viremia strata $(P<.001)$, and was characterized by a bell-shaped curve in which the highest prevalence was in the 1001-10 000 copies/mL stratum, with lower prevalence values at lower and higher viremia strata. Detection of resistance was consistent also at LLV. In particular, for viremia levels of 50-200 copies $/ \mathrm{mL}$, NRTI resistance was $41.3 \%$, NNRTI resistance was $40.2 \%$, unboosted PI resistance was $51.6 \%$, and PI/r resistance was $20.8 \%$. For viremia $201-500$ copies $/ \mathrm{mL}$, rates of resistance were $62.3 \%$, $69.3 \%, 30.8 \%$, and $28.0 \%$ respectively, which increased, for viremia $501-1000$ copies/mL, to $67.1 \%, 79.5 \%, 79.2 \%$, and $39.0 \%$ for each respective drug class (Table 3 ). Therefore, substantial levels of resistance can be detected also at LLV for all drug classes, with higher rates for NRTI and NNRTIs.

The distribution of drug resistance stratified for viremia was similar also considering samples only from patients failing their first-line regimen. In particular, a consistent proportion of NRTI and NNRTI resistance was found also at viremia levels 50-1000 copies/mL, whereas PI resistance was very low in samples from patients for whom a first-line PI/r-containing regimen was unsuccessful (for viremia 50-200 copies/mL: NRTI resistance, 19.2\%; NNRTI resistance, $13.6 \%$; PI/r resistance, 4.9\%; for viremia 201-500 copies/mL: NRTI resistance, $38.3 \%$; NNRTI resistance, $54.5 \%$; $\mathrm{PI} / \mathrm{r}$ resistance, $0 \%$; for viremia $501-$ 1000 copies/mL: NRTI resistance, 59.5\%; NNRTI resistance, 73.3\%; PI/r resistance, $7.1 \%)$.

The resistance to NRTI and NNRTI varied according to viremia strata also by restricting the analysis during 2008-2012, with a still-considerable prevalence of resistance in samples with viremia levels $\leq 1000$ copies/mL (Figure 2). By contrast, the prevalence of PI resistance was not influenced by viremia strata because it was very limited among all failures and was almost zero in patients failing their first-line $\mathrm{PI} / \mathrm{r}$-containing regimen.

Finally, by characterizing the prevalence of each single PRM in samples genotyped during the years 2008-2012, no major differences were found by analyzing samples with viremia $\leq 1000$ vs $>1000$ copies/mL (Supplementary Table 2). In particular, only the NNRTI PRM K103N was found with a significantly higher prevalence in patients who failed treatment with viremia $>1000$ copies $/ \mathrm{mL}(43.3 \%)$ vs $\leq 1000$ copies/mL $(20.2 \%)$ $(P<.001$, after multiple comparison correction).

\section{Virologic Outcome}

By Kaplan-Meier analysis, we found that the probability of reaching viremia $>1000$ copies/mL after LLV was significantly higher in patients with resistance than in those without resistance, as follows: at 24 weeks, $49.7 \%$ vs $4.2 \%$; at 48 weeks, $58.1 \%$ vs $8.7 \%$; at 72 weeks, $72.1 \%$ vs $15.2 \%(P<.001$; data not shown).

\section{DISCUSSION}

This study aimed at evaluating the reliability and usefulness of GRT in HIV-1-infected patients with detectable LLV, in a large data set of samples tested in 2 clinical centers in Italy. Our results showed that the genotyping success rate was $96 \%$ for the overall population. In particular, this success rate was very high also for viremia $>200$ copies $/ \mathrm{mL}$ (about $88 \%$ ), reaching about $93 \%$ at $501-1000$ copies $/ \mathrm{mL}$ and $>97 \%$ above 1000 copies $/ \mathrm{mL}$. Reasonable results in terms of success rate were obtained also for samples with viremia between 50 and 200 copies $/ \mathrm{mL}$. The ability to easily detect samples with LLV is mainly due to the improvement of the amplification step performed in our laboratories. The success of sequencing was very similar between $B$ and non-B strains, thus suggesting that the subtype diversity does not represent a limit. Our findings are in agreement with those recently obtained in other studies, showing a high success of amplification and sequencing also at $\operatorname{LLV}[16,19,21,26]$. Our results with LLV may not reflect the true population, but rather reflect founder effects, especially when nested amplification is needed. Nevertheless, phylogenetic analysis confirmed the reliability and reproducibility in our laboratories of genotypic tests at different viremia levels. Indeed, by evaluating 1613 pol sequences obtained from 470 patients with at least 2 GRTs performed at different times and with different viremia levels (ranging from $<50$ to $>100000$ copies $/ \mathrm{mL}$ ), very high similarity among sequences from the same patient was observed.

It should be emphasized that the additional step of the nested PCR does not affect the total cost of genotyping test, because the reagents used (Supplementary Methods) are inexpensive. Indeed, by adding the nested PCR step, the total amount of HIV-1 genotyping costs is increased only by about $€ 10-€ 15$ per sample performed. Therefore, we can conclude that the use of GRT for treatment optimization in HIV-1-infected patients with treatment failure at LLV is in any case cost effective.

The clinical relevance of our findings is related to the fact that in the last few years there has been an increased demand for GRTs for drug-experienced patients failing with LLV (mainly $\leq 500$ copies/mL, as shown in our analysis; Figure 1), explained by a greater tendency to closely monitor patients in terms of response to treatment and drug resistance. In our data set the proportion of requests from patients failing therapy with LLV has been about $30 \%$ since 2009 . 
Table 3. Drug Resistance Stratified by Plasma Viremia Ranges

\begin{tabular}{|c|c|c|c|c|c|c|c|c|c|c|c|c|c|c|c|c|c|c|c|c|c|c|c|c|c|}
\hline \multirow[b]{3}{*}{$\begin{array}{l}\text { Viremia Range, } \\
\text { Copies/mL }\end{array}$} & \multirow[b]{3}{*}{ No. } & \multicolumn{4}{|c|}{ All Samples } & \multicolumn{5}{|c|}{ Samples From Patients Taking NRTIs } & \multicolumn{5}{|c|}{ Samples From Patients Taking NNRTIs } & \multicolumn{5}{|c|}{$\begin{array}{l}\text { Samples From Patients Taking } \\
\text { Ritonavir-Boosted Pls }\end{array}$} & \multicolumn{5}{|c|}{$\begin{array}{l}\text { Samples From Patients Taking } \\
\text { Ritonavir-Unboosted Pls }\end{array}$} \\
\hline & & \multicolumn{4}{|c|}{ Resistance to Any Class } & \multirow[b]{2}{*}{ No. } & \multicolumn{4}{|c|}{ NRTI Resistance } & \multirow[b]{2}{*}{ No. } & \multicolumn{4}{|c|}{ NNRTI Resistance } & \multirow[b]{2}{*}{ No. } & \multicolumn{4}{|c|}{ PI Resistance } & \multirow[b]{2}{*}{ No. } & \multicolumn{4}{|c|}{ PI Resistance } \\
\hline & & $\%$ & $\begin{array}{c}P \\
\text { Value }^{\mathrm{a}}\end{array}$ & $\begin{array}{l}\text { PRMs, } \\
\text { No. }\end{array}$ & $\begin{array}{c}P \\
\text { Value }^{\mathrm{b}}\end{array}$ & & $\%$ & $\begin{array}{c}P \\
\text { Value }^{\mathrm{a}}\end{array}$ & $\begin{array}{l}\text { PRMs, } \\
\text { No. }\end{array}$ & $\begin{array}{c}P \\
\text { Value }^{\mathrm{b}}\end{array}$ & & $\%$ & $\begin{array}{c}P \\
\text { Value }^{\mathrm{a}}\end{array}$ & $\begin{array}{l}\text { PRMs. } \\
\text { No. }\end{array}$ & $\begin{array}{c}P \\
\text { Value }^{\mathrm{b}}\end{array}$ & & $\%$ & $\begin{array}{c}P \\
\text { Value }^{\mathrm{a}}\end{array}$ & $\begin{array}{l}\text { PRMs, } \\
\text { No. }\end{array}$ & $\begin{array}{c}P \\
\text { Value }^{\mathrm{b}}\end{array}$ & & $\%$ & $\begin{array}{c}P \\
\text { Value }^{\mathrm{a}}\end{array}$ & $\begin{array}{l}\text { PRMs, } \\
\text { No. }\end{array}$ & $\begin{array}{c}P \\
\text { Value }^{b}\end{array}$ \\
\hline Overall ranges & 3895 & 74.0 & & $3(0-7)$ & & 3761 & 66.0 & & $1(0-4)$ & & 1150 & 77.7 & & $2(1-2)$ & & 1977 & 40.5 & & $0(0-3)$ & & 632 & 61.7 & & $1(0-2)$ & \\
\hline $50-200$ & 396 & 52.8 & $<.001$ & $1(0-3)$ & $<.001$ & 385 & 41.3 & $<.001$ & $0(0-1)$ & $<.001$ & 82 & 40.2 & $<.001$ & $0(0-1)$ & $<.001$ & 255 & 20.8 & .842 & $0(0-0)$ & $<.001$ & 31 & 51.6 & .672 & $1(0-2)$ & $<.001$ \\
\hline $201-500$ & 287 & 70.0 & & $2(0-4)$ & & 273 & 62.3 & & $1(0-3)$ & & 75 & 69.3 & & $1(0-2)$ & & 168 & 28.0 & & $0(0-1)$ & & 26 & 30.8 & & $0(0-1)$ & \\
\hline $501-1000$ & 242 & 74.0 & & $3(0-6)$ & & 228 & 67.1 & & $1(0-4)$ & & 78 & 79.5 & & $1(1-2)$ & & 123 & 39.0 & & $0(0-2)$ & & 24 & 79.2 & & $1(1-2)$ & \\
\hline $1001-10000$ & 1102 & 86.1 & & $4(2-7)$ & & 1064 & 79.9 & & $2(1-4)$ & & 370 & 90.0 & & $2(1-3)$ & & 450 & 46.9 & & $0(0-3)$ & & 201 & 72.6 & & $2(0-3)$ & \\
\hline $10001-100000$ & 1212 & 76.7 & & $4(1-8)$ & & 1179 & 69.4 & & $2(0-4)$ & & 375 & 81.1 & & $2(1-2)$ & & 593 & 49.4 & & $0(0-4)$ & & 242 & 60.7 & & $1(0-2)$ & \\
\hline$>100000$ & 656 & 63.0 & & $2(0-8)$ & & 632 & 52.8 & & $1(0-4)$ & & 170 & 64.7 & & $1(0-2)$ & & 388 & 38.4 & & $0(0-3)$ & & 108 & 50.0 & & $0(0-2)$ & \\
\hline
\end{tabular}

The percentage of drug resistance and the median (IQR) number of PRMs were evaluated according to viremia ranges in 3895 patients with known therapeutic history and with at least 1 genotypic resistance test at failure. Genotypic resistance tests were performed between May 1999 and December 2012; median year (IQR) of genotyping was 2006 (2003-2009).

Resistance to an antiretroviral drug class was defined by the presence of at least 1 PRM included in the mutation list paneled by the International AIDS Society in 2013 [34], considering the NRTIs, NNRTIs, and PIs. In particular, we have defined (i) the resistance to any drug class in the overall samples analyzed; (ii) the resistance to NRTIs among samples from patients who received regimens that contained NRTIs; (iii) the resistance to NNRTIs among samples from patients who received regimens that contained NNRTIs; (iv) the resistance to Pls among samples from patients who received regimens that contained ritonavir-boosted Pls; and ( $v$ ) the resistance to PIs among samples from patients who received regimens that contained ritonavir-unboosted PIs.

In all the analyses performed, $P<.05$ was considered statistically significant.

Abbreviations: \%, proportion of samples with at least 1 PRM according to drug class; IQR, interquartile range; NNRTI, nonnucleos(t)ide reverse transcriptase inhibitor; NRTI, nucleos(t)ide reverse transcriptase inhibitor $\mathrm{Pl}$, protease inhibitor; PRM, primary resistance mutation.

a Potential differences in the percentage of resistance among the different viremia ranges were evaluated by the $\chi^{2}$ test for trend

${ }^{b}$ Potential differences in the number of PRMs among the different viremia ranges were evaluated by the Kruskal-Wallis test. 


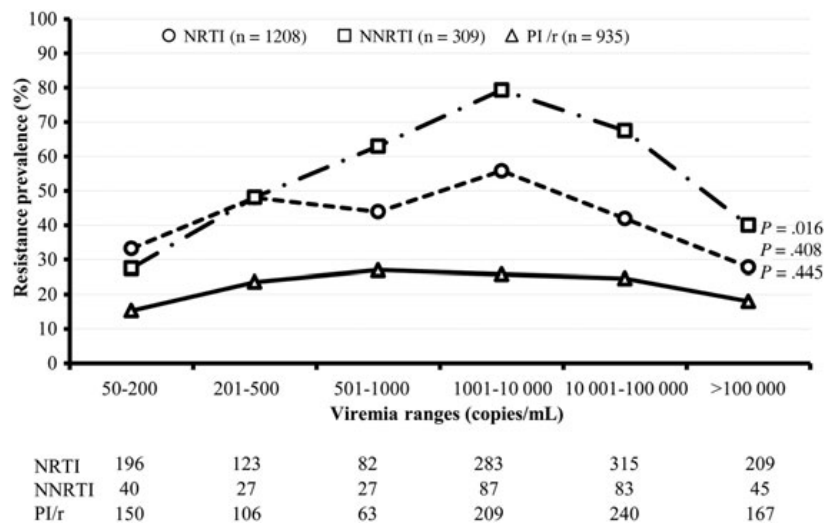

Figure 2. Resistance to nucleos(t)ide reverse transcriptase inhibitor (NRTI), nonnucleos(t)ide reverse transcriptase inhibitor (NNRTI), or ritonavir-boosted protease inhibitor $(\mathrm{Pl} / \mathrm{r}$ ) classes in samples collected from January 2008 to December 2012 stratified for plasma viremia ranges. Analysis was performed on 1317 samples from patients for whom NRTI, NNRTI, or ritonavir-boosted PI treatment failed. Resistance was defined as the presence of at least 1 primary NRTI, NNRTI, or PI resistance mutation among those paneled by the International AIDS Society-USA [34]. Potential differences in the percentage of resistance among the different viremia ranges were evaluated by $\chi^{2}$ test for trend. $P<.05$ was considered significant.

Moreover, our results corroborate the already discussed recruitment about drug resistance presence also at viremia levels $\leq 1000$ copies/mL [26, 35-37], underlining the importance of GRTs also at LLV for the optimization of therapy in patients under virologic failure. In this regard, it should be emphasized that the optimization of the sequencing protocol in the last years has led to a higher accuracy in detecting the PRMs for each viremia level. In our study, a considerable prevalence of resistance was found also at LLV among the samples analyzed from patients for whom therapy was unsuccessful. This finding proves that the detection of drug resistance is not a rare event in these low viremia ranges.

A decline in the prevalence of PRMs was observed also at the very high viremia strata among drug-experienced individuals. This decline is likely to reflect suboptimal medication adherence, with lower drug resistance selection [35].

A considerable prevalence of resistance to NRTIs and NNRTIs at LLV was found also when the analysis was restricted to 1317 samples from patients failing therapy in the last few years. This prevalence can be due to the large usage of lowgenetic-barrier drugs such as lamivudine/emtricitabine or efavirenz/nevirapine. By the evaluation of the effect of LLV resistance on subsequent virologic outcome, we found that the probability of reaching viremia $>1000$ copies $/ \mathrm{mL}$ by 72 weeks after LLV was significantly higher in patients with resistance than in those without resistance. This strongly suggests that the early detection of resistance (when viremia is still $<1000$ copies $/ \mathrm{mL}$ ) may prevent the evolution toward (i) a virologic failure with higher viremia and (ii) the accumulation of additional mutations, thus affecting the choice of future therapeutic regimens. A potential limitation of this analysis could be it that was performed only on a very small data set of patients. In line with our data, a recent study, performed in a larger cohort of patients, confirmed that LLV resistance is predictive of subsequent virologic failure [37]. Taken together, these results reinforce the concept that GRT may be useful in the management of failure even at LLV.

Data presented in our study, in agreement with previous articles $[35,36]$ and with data recently presented $[26,37,38]$, suggest that newer guidelines may reconsider the importance of GRT in clinical practice even at LLV. Indeed, despite the technical improvements achieved in the last few years, treatment guidelines still do not usually recommend GRT in patients with a plasma viral load ranging between 50 and 1000 copies/mL $[2,4]$.

In conclusion, our study, carried out in standard clinical practice, confirms that drug resistance mutations can be detected even at low viral load, regardless of the antiretroviral target genes, and can remarkably reduce the current therapeutic options for further regimens. Our findings emphasize the importance of using the genotypic test at the first treatment failures even at low viremia, to guide the choice of an effective alternative regimen.

\section{Supplementary Data}

Supplementary materials are available at Clinical Infectious Diseases online (http://cid.oxfordjournals.org/). Supplementary materials consist of data provided by the author that are published to benefit the reader. The posted materials are not copyedited. The contents of all supplementary data are the sole responsibility of the authors. Questions or messages regarding errors should be addressed to the author.

\section{Notes}

Acknowledgments. We gratefully thank Andrea Biddittu, Massimiliano Bruni, Luca Carioti, Fabio Continenza, Alberto Giannetti, Massimo Giuliani, Anna Pacifici, Daniele Pizzi, and Marzia Romani for sequencing and data management, together with all the members of the Resistance Group of the National Institute for Infectious Diseases "Lazzaro Spallanzani": R. Acinapura, A. Ammassari, A. Antinori (Co-Chair), G. Anzidei, F. Baldini, R. Bellagamba, E. Boumis, F. Ceccherini-Silberstein, S. Cerilli, R. D’Arrigo, P. De Longis, G. D’Offizi, F. Forbici, L. Fabeni, S. Galati, M. L. Giancola, E. Girardi, C. Gori, R. Libertone, G. Liuzzi, P. Lorenzini, P. Marconi, S. Mosti, P. Narciso, V. Neri, E. Nicastri, C. F. Perno (CoChair), C. Pinnetti, M. M. Santoro, P. Sette, V. Svicher, C. Tommasi, V. Tozzi, U. Visco-Comandini, and M. Zaccarelli, and the Resistance Group of University Hospital Tor Vergata: C. Alteri, M. Andreoni (CoChair), D. Armenia, A. Bertoli, A. R. Buonomini, F. Ceccherini-Silberstein, L. Dori, S. Giannella, T. Guenci, G Maffongelli, C. F. Perno (Co-Chair), M. Pollicita, R. Salpini, M. M. Santoro, L. Sarmati, V. Svicher.

Financial support. This work was supported by the European Commission Framework 7 Programme (CHAIN, the Collaborative HIV and AntiHIV Drug Resistance Network, Integrated Project number 223131); the European AIDS Treatment Network (NEAT, contract number LSHT/CT/ 
2006/037570); the Italian Ministry of Health (CUP: E81J10000000001, Ricerca Corrente and Progetto AIDS, grant number 40H78); and the AVIRALIA Foundation (unrestricted grant).

Potential conflicts of interest. M. M. S. has received funds for attending symposia, speaking, and organizing educational activities from Abbott, Bristol-Myers Squibb (BMS), Merck Sharp \& Dohme (MSD), and Janssen. A. Am. has received funds for advisory board membership from MSD. E. N. has received funds for attending lectures (including services on speakers' bureaus) and grant research support from Janssen, Pfizer, MSD, and ViiV Healthcare. N. P. has received funds for attending lectures from Pfizer, Novartis, MSD, Astellas, Carefusion, and Johnson \& Johnson. M. A. has received funds for attending symposia, speaking, and organizing educational activities as well as grant research support from Abbvie, BMS, Gilead Sciences, and ViiV Healthcare. F. C.-S. has received funds for attending symposia, speaking, and organizing educational activities from Abbott, MSD, Gilead, Janssen, ViiV Healthcare, Roche, and Virco. A. An. has received funds for attending symposia, speaking, grant research support, and consultancy from Abbvie, BMS, Gilead Sciences, MSD, Janssen, and ViiV Healthcare. C. F. P. has received funds for attending symposia, speaking, organizing educational activities, grant research support, consultancy, and advisory board membership from Abbott, BMS, Gilead, MSD, Janssen, Pfizer, Roche, and ViiV Healthcare. All other authors report no potential conflicts.

All authors have submitted the ICMJE Form for Disclosure of Potential Conflicts of Interest. Conflicts that the editors consider relevant to the content of the manuscript have been disclosed.

\section{References}

1. Palella FJ Jr, Delaney KM, Moorman AC, et al. Declining morbidity and mortality among patients with advanced human immunodeficiency virus infection. HIV Outpatient Study Investigators. N Engl J Med 1998; 338:853-60.

2. European AIDS Clinical Society Guidelines (EACS). Guidelines for the clinical management and treatment of HIV infected adults in Europe. Version 7.0, October 2013. Available at: http://www.eacsociety.org/ Portals/0/Guidelines_Online_131014.pdf. Accessed 22 July 2013.

3. Thompson MA, Aberg JA, Hoy JF, et al. Antiretroviral treatment of adult HIV infection: 2012 recommendations of the International Antiviral Society-USA panel. JAMA 2012; 308:387-402.

4. Guidelines for the use of antiretroviral agents in HIV-1-infected adults and adolescents. February 2013. Available at: http://www.aidsinfo.nih. gov/ContentFiles/AdultandAdolescentGL.pdf. Accessed 22 July 2013.

5. Lederman MM, Valdez H. Immune restoration with antiretroviral therapies: implications for clinical management. JAMA 2000; 284:223-8.

6. Gutierrez F, Padilla S, Masiá M, et al. Clinical outcome of HIV-infected patients with sustained virologic response to antiretroviral therapy: long-term follow-up of a multicenter cohort. PLoS One 2006; 1:e89.

7. Moore RD, Keruly JC, Bartlett JG. Improvement in the health of HIVinfected persons in care: reducing disparities. Clin Infect Dis 2012; 55:1242-51.

8. Geretti AM, Harrison L, Green H, et al. Effect of HIV-1 subtype on virologic and immunologic response to starting highly active antiretroviral therapy. Clin Infect Dis 2009; 48:1296-305.

9. Cozzi-Lepri A, Mussini C, Bonora S, et al. The chance of suppressing viral load $\leq 80$ copies $/ \mathrm{ml}$ according to initial viral load and type of regimen started: an observational study. In: 13th European AIDS Conference/European AIDS Clinical Society Guidelines (EACS). Belgrade, Serbia, 12-15 October 2011. Abstract PE7.7/3.

10. Santoro MM, Armenia D, Alteri C, et al. Impact of pre-therapy viral load on virological response to modern first-line HAART. Antiv Ther 2013. doi:10.3851/IMP2531.

11. Gunthard HF, Wong JK, Ignacio CC, et al. Human immunodeficiency virus replication and genotypic resistance in blood and lymph nodes after a year of potent antiretroviral therapy. J Virol 1998; 72:2422-8.
12. Parkin NT, Deeks SG, Wrin MT, et al. Loss of antiretroviral drug susceptibility at low viral load during early virological failure in treatmentexperienced patients. AIDS 2000; 14:2877-87.

13. Aleman S, Soderbarg K, Visco-Comandini U, Sitbon G, Sonnerborg A. Drug resistance at low viraemia in HIV-1-infected patients with antiretroviral combination therapy. AIDS 2002; 16:1039-44.

14. Delaugerre C, Gallien S, Flandre P, et al. Impact of low-level-viremia on HIV-1 drug-resistance evolution among antiretroviral treated-patients. PLoS One 2012; 7:e36673. doi:10.1371/journal.pone.0036673.

15. Vandamme AM, Camacho RJ, Ceccherini-Silberstein F, et al. European HIV Drug Resistance Guidelines Panel. European recommendations for the clinical use of HIV drug resistance testing: 2011 update. AIDS Rev 2011; 13:77-108.

16. Mackie N, Dustan S, McClure MO, et al. Detection of HIV-1 antiretroviral resistance from patients with persistently low but detectable viraemia. J Virol Methods 2004; 119:73-81.

17. Nettles RE, Kieffer TL, Simmons RP, et al. Genotypic resistance in HIV1 -infected patients with persistently detectable low-level viremia while receiving highly active antiretroviral therapy. Clin Infect Dis 2004; 39:1030-7.

18. Gale HB, Kan VL, Shinol RC. Performance of the TruGene human immunodeficiency virus type 1 genotyping kit and OpenGene DNA sequencing system on clinical samples diluted to approximately 100 copies per milliliter. Clin Vaccine Immunol 2006; 13:235-8.

19. Waters L, Mandalia S, Asboe D. Successful use of genotypic resistance testing in HIV-1-infected individuals with detectable viraemia between 50 and 1000 copies/mL. AIDS 2006; 20:778-9.

20. Mitsuya Y, Winters MA, Fessel WJ, et al. HIV-1 drug resistance genotype results in patients with plasma samples with HIV-1 RNA levels less than 75 copies/mL. J Acquir Immune Defic Syndr 2006; 43:56-9.

21. Cane PA, Kaye S, Smit E, et al. Genotypic antiretroviral drug resistance at low viral loads in the UK. HIV Med 2008; 9:673-6.

22. Yang Z, Morrison R, Oates C, et al. HIV-1 genotypic resistance testing on low viral load specimens using the Abbott ViroSeq HIV-1 genotyping system. Lab Med 2008; 39:671-3.

23. Pattery T, De Wolf H, Verlinden Y, et al. Efficiency of HIV-1 PR-RT genotyping on clinical isolates with viral load less than 1000 copies/ mL: a 12 year analysis. In: 8th European HIV Drug Resistance Workshop, Sorrento, Italy, 2010. Poster 1.

24. Milia MG, Allice T, Gregori G, et al. Magnetic-silica based nucleic acid extraction for human immunodeficiency virus type-1 drug-resistance testing in low viremic patients. J Clin Virol 2010; 47:8-12.

25. Stelzl E, Troppan KT, Winkler M, et al. Optimized protocol for detection of HIV-1 drug mutations in patients with low viral load. J Virol Methods 2010; 168:152-4.

26. Gonzalez-Serna A, Min JE, Woods C, Li J, Harrigan PR, Swenson LC. Low level HIV viremia and drug resistance testing. In: 20th Program and abstracts of the 20th Conference on Retroviruses and Opportunistic Infections, Atlanta, 2013. Poster 601.

27. Ruelle J, Jnaoui K, Lefèvre I, et al. Comparative evaluation of the VERSANT HIV-1 RNA 1.0 kinetic PCR molecular system (kPCR) for the quantification of HIV-1 plasma viral load. J Clin Virol 2009; 44:297-301.

28. Paba P, Fabeni L, Ciccozzi M, et al. Performance evaluation of the COBAS/TaqMan HIV-1 v2.0 in HIV-1 positive patients with low viral load: a comparative study. J Virol Methods 2011; 173:399-402.

29. Sire JM, Vray M, Merzouk M, et al. Comparative RNA quantification of HIV-1 group M and non-M with the Roche Cobas AmpliPrep/Cobas TaqMan HIV-1 v2.0 and Abbott Real-Time HIV-1 PCR assays. J Acquir Immune Defic Syndr 2011; 56:239-43.

30. Ceccherini-Silberstein F, Gago F, Santoro M, et al. High sequence conservation of human immunodeficiency virus type 1 reverse transcriptase under drug pressure despite the continuous appearance of mutations. J Virol 2005; 79:10718-29.

31. Mukaide M, Sugiura W, Matuda M, et al. Evaluation of ViroSeq-HIV version 2 for HIV drug resistance. Jpn J Infect Dis 2000; 53:203-5. 
32. Cunningham S, Ank B, Lewis D, et al. Performance of the Applied Biosystems ViroSeq human immunodeficiency virus type 1 (HIV-1) genotyping system for sequence-based analysis of HIV-1 in pediatric plasma samples. J Clin Microbiol 2001; 39:1254-7.

33. Kimura M. A simple method for estimating evolutionary rate of base substitutions through comparative studies of nucleotide sequences. J Mol Evol 1980; 16:111-20.

34. Johnson VA, Calvez V, Gunthard HF, et al. Update of the drug resistance mutations in HIV-1: March 2013. Top Antivir Med 2013; 21:6-14.

35. Mackie NE, Phillips AN, Kaye S, et al. Antiretroviral drug resistance in HIV-1-infected patients with low-level viremia. J Infect Dis 2010; 201:1303-7.

36. Prosperi MC, Mackie N, Di Giambenedetto S, et al. Detection of drug resistance mutations at low plasma HIV-1 RNA load in a
European multicentre cohort study. J Antimicrob Chemother 2011; 66: 1886-96.

37. Swenson LC, Gonzalez-Serna A, Min J, Woods CK, Li JZ, Harrigan PR. HIV drug resistance occurring during low-level viraemia is associated with subsequent virological failure. Abstract 32. Program and abstracts of the International Workshop on HIV \& Hepatitis Virus Drug Resistance and Curative Strategies (Toronto, Canada). Antiviral Ther 2013; 18(suppl 1):A40.

38. Assoumou L, Descamps D, Yerly S, et al. Prevalence of HIV-1 drug resistance in treated patients with viral load $>50$ copies $/ \mathrm{mL}$ in 2009: a French nationwide study. J Antimicrob Chemother 2013; 68: 1400-05.

39. Hochberg Y, Benjamini Y. More powerful procedures for multiple significance testing. Stat Med 1990; 9:811-8. 